\title{
Histerectomía obstétrica en el Hospital General Dr. Aurelio Valdivieso, Oaxaca, México
}

\author{
Omar Calvo Aguilar ${ }^{1}$, Esther Rosas Carvallar ${ }^{2}$, Javier Vásquez Martínez 3 , Jenny \\ Hernández Cuevas. 4 \\ ${ }^{1}$ Médico Especialista en Ginecología y Obstetricia, Especialista en Medicina Materno Fetal, Maestro en Ciencias Médicas. \\ ${ }^{2}$ Residente de Tercer año en Ginecología y Obstetricia. ${ }^{3}$ Residente de Cuarto año en Ginecología y Obstetricia. ${ }^{4}$ Médica \\ Especialista en Ginecología y Obstetricia., Directora del Hospital General "Dr. Aurelio Valdivieso". Hospital General, "Dr. \\ Aurelio Valdivieso", Oaxaca, México.
}

\section{RESUMEN}

Objetivo: Determinar la incidencia, principales indicaciones y complicaciones de la histerectomía obstétrica (HO) en un hospital de segundo nivel de atención a cuatro años de implementar el programa de prevención y manejo de la hemorragia obstétrica. Método: Estudio de tipo descriptivo de revisión de expedientes clínicos, de tipo transversal, analítico y retrospectivo. Resultados: Se hallaron 51 expedientes de pacientes a quiénes se realizó HO de enero de 2012 a noviembre de 2015. La prevalencia fue de 17,1/10.000 nacimientos, la incidencia por año fue de 1,7 (2012), 1,7 (2013), 1,4 (2014) y 1,9 (2015) por cada mil nacimientos respectivamente. La prevalencia de $\mathrm{HO}$ post-cesárea fue de 25,6/10.000 y en el post-parto de 10,6/10.000. Las variables que alcanzaron significancia entre cirugía programada y de emergencia fue pérdida sanguínea y necesidad de transfusiones sanguíneas. El procedimiento se asocia a anemia en el puerperio 7 veces más y las principales indicaciones para realizar el procedimiento fueron alteración de la adherencia placentaria e hipotonía. Conclusiones: El diagnóstico prenatal de anomalías en la adherencia placentaria, la mejor utilización de hemoderivados y la técnica quirúrgica ha eliminado la mortalidad materna por hemorragia obstétrica masiva en los últimos cuatro años en el Hospital General Dr. Aurelio Valdivieso.

\section{PALABRAS CLAVE: Histerectomía obstétrica, hemorragia obstétrica, adherencia placentaria anormal, atonía uterina, cesárea}

\section{SUMMARY}

Objective: To determine the incidence, main indications and complications of obstetric hysterectomy in a secondary hospital care to four years to implement the program of prevention and management of obstetric hemorrhage. Methods: Descriptive study of review of clinical records, transversal, analytical and retrospective. Results: 51 cases of patients who obstetric hysterectomy $(\mathrm{OH})$ was held between January 2012 to November 2015. The prevalence was 17.1/10,000. The incidence per year was 1.7 (2012), 1.7 (2013), 1.4 (2014) and 1.9 (2015) per 1000 births, respectively. The prevalence of post-cesarean $\mathrm{OH}$ was 25.6/10,000 while postpartum $\mathrm{OH}$ was $10.6 / 10,000$. The variables that reached significance between scheduled and emergency surgery was blood loss and need for blood transfusions. The procedure is associated with anemia in the postpartum period 7 times and the main indications for the procedure were alteration abnormal placental adhesion and uterine atony. Conclusions: The prenatal diagnosis of abnormal placental adhesion, 
better use of blood products and surgical technique has eliminated maternal mortality by massive obstetric hemorrhage in the last four years in the General Hospital Dr. Aurelio Valdivieso.

\section{KEY WORDS: Obstetric hysterectomy, obstetric hemorrhage, abnormal placental adhesion, uterine atony, cesarean}

\section{INTRODUCCIÓN}

La histerectomía obstétrica $(\mathrm{HO})$ es una intervención quirúrgica que en la actualidad se encuentra reservada sólo para aquellas situaciones en las que el manejo conservador para preservar el útero y la función reproductiva han fallado, considerándose el procedimiento como una intervención indicada en los casos de emergencia obstétrica para preservar la vida (1).

El procedimiento no es de primera elección debido a las altas tasas de morbilidad quirúrgica que presenta por eventos adversos previsibles, reportándose altas tasas de morbilidad desde el inicio de su práctica en el siglo pasado, secundario a procesos infecciosos por falta de antibióticos y técnicas de asepsia (2). El procedimiento se realiza de forma segura hasta la segunda mitad del siglo anterior, llegando a tener una prevalencia entre 0,24 hasta 1,4/1000 nacidos vivos (2). La evolución que ha presentado la histerectomía obstétrica desde hace cinco décadas al día de hoy es variable, con alta prevalencia en sus inicios $(0,85 / 1000)$, hasta en esta última etapa un incremento de manera gradual a raíz de los casos de acretismo placentario $(0,40 / 1000)(2)$.

Dentro de las principales indicaciones para realizar la $\mathrm{HO}$ se encuentra la hemorragia obstétrica por atonía uterina, las laceraciones del útero y los procesos infecciosos, pero sin duda es la hemorragia por atonía la principal indicación (3). El procedimiento tiene alta morbilidad cuando la hemorragia es secundaria a adherencia anómala de la placenta, a placenta previa o desprendimiento prematuro de placenta normo inserta, principalmente por choque hipovolémico o séptico, coagulación intravascular diseminada y lesiones de vías urinarias. Las complicaciones de menor gravedad que aumentan la estancia hospitalaria y generan carga económica para el sistema son: complicaciones febriles, hematomas y/o hemorragia de cúpula vaginal, hematuria, íleo intestinal, neumonía, pulmón de choque, hematomas, abscesos pélvicos y de pared abdominal, insuficiencia renal, infección urinaria y disturbios psicológicos (4).

En el Hospital General de segundo nivel de los Servicios de Salud de Oaxaca en la capital estatal, hasta el año 2011 se habían presentado entre tres y cuatro muertes maternas por hemorragia obstétrica masiva anuales, situación por la que se hizo necesario establecer el programa del manual código rojo OMS/OPS (5), adaptado a esta unidad hospitalaria, estableciéndose el manejo quirúrgico programado para evitar pérdida de sangre, complicaciones anestésicas, menor ingreso a las áreas de cuidados intensivos y disminución en el consumo de hemoderivados, ventajas que se han observado cuando el procedimiento se realiza de forma programada sea cesárea-histerectomía o histerectomía en bloque $(6,7)$. Oaxaca es uno de los dos estados que de acuerdo con el informe ejecutivo de gobierno del año 2015 disminuyó en $75 \%$ la mortalidad materna, así como también la brecha con respecto a la esperada de acuerdo con el $5^{\circ}$ Objetivo de Desarrollo del Milenio, saliendo de los primeros lugares en los últimos cuatro años (8).

La histerectomía post cesárea es el procedimiento más realizado durante la etapa puerperal, considerándola un indicador de calidad de la atención obstétrica, porque se asocia de manera substancial con la morbilidad obstétrica extrema.

El objetivo de este estudio fue determinar la incidencia, principales indicaciones y complicaciones de la $\mathrm{HO}$ en un hospital de segundo nivel de atención, luego de la implementación del programa de prevención y manejo de la Hemorragia Obstétrica Masiva en el año 2011.

\section{PACIENTES Y MÉTODO}

A partir del año 2011 se estableció el protocolo de manejo para emergencias obstétricas, mediante el cual se capacitó al personal en el manejo de la hemorragia obstétrica masiva, en dicho programa se utilizó la propuesta del manual de la OMS "código rojo" (5), además de establecer nuevos protocolos en el manejo quirúrgico de la histerectomía obstétrica mediante diagnóstico prenatal de alteraciones en la adherencia placentaria por ultrasonido. Durante la implementación del programa se mantuvo disponible personal capacitado en manejo quirúrgico de la hemorragia obstétrica, fármacos uterotónicos, hemoderivados y disponibilidad de quirófano las 24 horas.

Se llevó a cabo un estudio observacional, transversal, retrospectivo y descriptivo, del 1 de enero 
del 2012 al 30 Noviembre del 2015, en el que se revisaron los expedientes pacientes a quienes se les practicó histerectomía por causa obstétrica, de cualquier edad y en cualquier momento de la gestación. Se estimó la tasa de incidencia de histerectomía obstétrica la cual fue catalogada en función de emergencia o programada, para el primer rubro se contempló: histerectomía poscesárea (no programada), histerectomía posparto, histerectomía poscuretaje o poslegrado; en el segundo rubro se incluyó a la cesárea-histerectomía programada (C-H) e histerectomía en bloque. Se identificaron las características clínicas, sociodemográficas, indicaciones y complicaciones, las cuales se expresaron como frecuencias, porcentajes y medidas de tendencia central. Se realizó contraste de hipótesis de las variables cuantitativas mediante prueba de $t$ considerándola significativa con un valor $p<0,05$. El análisis estadístico se realizó el paquete SPSS 22.

\section{RESULTADOS}

Durante el periodo de búsqueda fueron hallados 51 expedientes que correspondieron a $\mathrm{HO}$ dentro de un total de 29.791 expedientes de procedimientos obstétricos atendidos con recién nacido vivo (NV) de enero de 2012 a noviembre de 2015, con una prevalencia de 17,1/10.000 NV en el periodo evaluado. De este grupo de $\mathrm{HO}$ se programaron C-H 22 casos, considerándose como procedimiento de emergencia en 29 casos. En 33 de los casos se practicó la HO luego de una cesárea y 18 casos fueron posterior a parto. La prevalencia de $\mathrm{HO}$ post-cesárea fue de $25,6 / 10.000 \mathrm{NV}$, en tanto que en el postparto fue de 10,6/10.000 NV. Las características de los nacimientos por año y acumulados se encuentran descritos en la Tabla I.

La tendencia observada de la $\mathrm{HO}$ en la institución se mantiene constante, la incidencia por año fue de 1,7 (2012), 1,7 (2013), 1,4 (2014) y 1,9 (2015) por cada mil nacimientos respectivamente, con incidencia para el periodo de 17,1/10.000 NV, destacando que la incidencia luego del nacimiento por cesárea es más alta con respecto al nacimiento por parto con una razón de 1,8 veces más.

En cuanto a la descripción del grupo de pacientes que fue sometido a este procedimiento se encontró que la edad promedio fue de 31,3 \pm 4,6 años. La edad máxima reportada fue de 41 años y la mínima de 18 años. Ocho casos ocurrieron en el grupo de 20-24 años, 15 casos en el grupo de 2529, 18 casos en el grupo de 30-34 años, 9 casos en el grupo de 35 y más años, en adolescentes hubo solo 1 caso. Respecto al estado civil, aproximadamente el $80 \%$ de las mujeres era casada o en unión libre, sólo nueve de los casos reportaron ser solteras. El resto de las características de la población se encuentran resumidas en la Tabla II.

La indicación principal para realizar la $\mathrm{HO}$ fue el diagnóstico de anomalías de la adherencia placentaria en cualquiera de sus variedades (acreta, increta, percreta), seguida por los trastornos del tono uterino con 17 casos, ambas entidades representaron más del $70 \%$ de los casos de HO. El diagnóstico de sepsis fue el menor en orden presentación con 1 caso. El resto de los diagnósticos se presentan en la Tabla III.

\section{Tabla I \\ CARACTERÍSTICAS POR AÑO DE LOS NACIMIENTOS Y PROCEDIMIENTOS QUIRÚRGICOS EN EL HOSPITAL GENERAL “DR. AURELIO VALDIVIESO” 2012-2015}

\begin{tabular}{ccccccccc}
\hline Año & $\begin{array}{c}\text { Nacidos } \\
\text { vivos }\end{array}$ & Cesáreas & Parto & $\begin{array}{c}\text { Índice de } \\
\text { cesáreas } \\
(\%)\end{array}$ & $\begin{array}{c}\mathrm{HO} \\
(\mathrm{n})\end{array}$ & $\begin{array}{c}\mathrm{HO} \\
(\mathrm{x} 1000 \mathrm{NV})\end{array}$ & $\begin{array}{c}\mathrm{HO} \\
\mathrm{P} / \mathrm{ces} \text { (cea } \\
(\mathrm{n})\end{array}$ & $\begin{array}{c}\mathrm{HO} \\
\mathrm{P} / \mathrm{parto} \\
(\mathrm{n})\end{array}$ \\
\hline 2012 & 6.859 & 3.063 & 3.796 & 44.65 & 12 & 1,74 & 8 & 4 \\
2013 & 7.985 & 3.268 & 4.717 & 40.92 & 14 & 1,75 & 10 & 4 \\
2014 & 7.668 & 3.241 & 4.427 & 42.26 & 11 & 1,43 & 6 & 5 \\
2015 & 7.279 & 3.348 & 3.931 & 45.99 & 14 & 1,92 & 9 & 5 \\
\hline Total & 29.791 & 12.920 & 16.871 & 43.36 & 51 & 1,71 & 33 & 18 \\
\hline
\end{tabular}

Fuente: Archivo de Estadística Hospital General "Dr. Aurelio Valdivieso”. HO: histerectomía obstétrica. NV: nacidos vivos. P: pos. 
Tabla II

CARACTERÍSTICAS DEL GRUPO DE PACIENTES INTERVENIDAS POR HISTERECTOMÍA OBSTÉTRICA

\begin{tabular}{lcc}
\hline & Media & $\pm \mathrm{DE}$ \\
\hline Edad (años) & 31,3 & 4,62 \\
Gestaciones (n) & 3,43 & 1,28 \\
Partos previos (n) & 0,82 & 1,21 \\
Cesáreas previas (n) & 1,35 & 0,91 \\
Amenorrea (semanas) & 36,19 & 2,60 \\
\hline
\end{tabular}

Fuente: Archivo de Estadística Hospital General "Dr. Aurelio Valdivieso".

Tabla III

PRINCIPALES DIAGNÓSTICOS DE LOS CASOS DE HISTERECTOMÍA OBSTÉTRICA

\begin{tabular}{lcc}
\hline Diagnóstico & $\mathrm{n}$ & $\%$ \\
\hline Sepsis & 1 & 1,96 \\
Hipo/atonía uterina & 17 & 33,33 \\
Cesárea iterativa & 3 & 5,88 \\
Anomalía adherencia placentaria & 20 & 39,21 \\
Placenta previa & 6 & 11,76 \\
Rotura uterina & 2 & 3,92 \\
Desgarro & 2 & 3,92 \\
\hline Total & 51 & $100 \%$ \\
\hline
\end{tabular}

Fuente: Archivo de Estadística Hospital General "Dr. Aurelio Valdivieso".

\section{Tabla IV \\ VARIABLES CUANTITATIVAS EVALUADAS EN PACIENTES CON HISTERECTOMÍA OBSTÉTRICA}

\begin{tabular}{lcc}
\hline Variables & Media & $\pm \mathrm{DE}$ \\
\hline Pérdida sanguínea $(\mathrm{ml})$ & $1.993,47$ & $1.123,65$ \\
Hemoglobina previa a cirugía $(\mathrm{g} / \mathrm{dl})$ & 11,78 & 1,96 \\
Hemoglobina control $(\mathrm{g} / \mathrm{dl})^{\star}$ & 9,37 & 1,73 \\
Tiempo quirúrgico (min) & 114,06 & 31,26 \\
Días de estancia & 6,75 & 5,75 \\
Días en UCl & 0,86 & 1,51 \\
Concentrado eritrocitario & 2,96 & 2,4 \\
(\# paquetes) & & \\
\hline
\end{tabular}

Fuente: Archivo de Estadística Hospital General "Dr. Aurelio Valdivieso". UCl: Unidad de Cuidados Intensivos. * 12-24 horas poscirugía.
Antes de iniciar el procedimiento quirúrgico se observó que 6 pacientes cursaron con anemia, 2 de las cuales presentaron cuadro severo. Luego de realizarse el procedimiento quirúrgico se observó que 26 pacientes presentaron anemia de las cuales 6 fueron casos severos. La ocurrencia observada para presentar anemia por el evento quirúrgico es 7,8 veces más (IC $95 \% 2,83-21,49$ ), en tanto que para anemia severa es de 2,09 veces más (IC 95\% $0,36-11,93)$. La hemoglobina cuantificada promedio antes del procedimiento quirúrgico fue de $11,78 \pm 1,96 \mathrm{~g}$. La hemoglobina media cuantificada luego del procedimiento quirúrgico fue 9,37 \pm $1,73 \mathrm{~g}$. El cuadro de hemorragia obstétrica masiva considerada con pérdida mayor a $1.200 \mathrm{ml}$ durante el procedimiento quirúrgico fue de 33 casos, de estas requirieron transfusión sanguínea 29 casos con una media de paquetes transfundidos de 3,28 $\pm 3,3$ concentrados eritrocitarios; la pérdida media reportada de sangre fue de $1.943,17 \pm 1.123,65 \mathrm{ml}$ (Tabla IV).

El procedimiento quirúrgico se realizó en aproximadamente 114,06 $\pm 31,26$ min. El tiempo quirúrgico mínimo reportado para el procedimiento fue de 60 min y el máximo fue de $200 \mathrm{~min}$. Pasado el procedimiento quirúrgico, sólo 17 pacientes fueron ingresadas al área de terapia intensiva, y los días de estancia promedio postquirúrgica fueron de 0,86 $\pm 1,2$ días, en tanto que los días promedio de hospitalización fue de 5,75 $\pm 6,75$ días. La paciente con mayor permanencia en hospitalización fue de 18 días y 6 pacientes que tuvieron la menor estancia con 2 días.

Las complicaciones observadas durante hospitalización se presentaron en 9 casos distribuidos de la siguiente manera: 3 casos presentaron preeclampsia, 4 complicaciones infecciosas, 1 hematoma en la herida quirúrgica y 1 con paro cardiorrespiratorio que revirtió con maniobras durante el transoperatorio.

Finalmente de acuerdo con la clasificación del procedimiento si este fue realizado de manera programada por contar con diagnóstico prenatal, o bien si fue realizado como una emergencia, se llevó a cabo una comparación por contraste de hipótesis para determinar si la pérdida sanguínea del procedimiento programado, era significativamente menor, además se compararon otras variables como edad de las pacientes, pérdida sanguínea calculada (hemorragia), cuantificación de hemoglobina previa al procedimiento y control a las $12-24$ horas, y los días de estancia hasta su egreso hospitalario. Los resultados del contraste de hipótesis solo mostraron diferencias significativas entre mayor pérdida sanguínea y mayor necesidad de transfusiones en la $\mathrm{HO}$ de urgencia (Tabla V). 
Tabla V CONTRASTE DE HIPÓTESIS DE PROCEDIMIENTOS DE URGENCIA VS PROGRAMADA

\begin{tabular}{|c|c|c|c|c|c|c|}
\hline \multirow[b]{2}{*}{ Variable } & \multicolumn{2}{|c|}{$\begin{array}{c}\text { HO urgencia } \\
n=29\end{array}$} & \multicolumn{2}{|c|}{$\begin{array}{c}\mathrm{HO} \text { programada } \\
\mathrm{n}=22\end{array}$} & \multirow[b]{2}{*}{ Valor $\mathrm{p}$} & \multirow[b]{2}{*}{ IC 95\% } \\
\hline & Media & $\pm \mathrm{DE}$ & Media & $\pm \mathrm{DE}$ & & \\
\hline Edad (años) & 31,95 & 5,65 & 30,96 & 5,48 & 0,82 & $-1,92 / 3,31$ \\
\hline Amenorrea (sem) & 36,29 & 2,34 & 36,08 & 2,89 & 0,21 & $-1,27 / 1,68$ \\
\hline Gestaciones (n) & 3,42 & 1,41 & 3,44 & 1,15 & 0,23 & $-0,74 / 0,71$ \\
\hline Partos (n) & 0,85 & 1,31 & 0,76 & 1,26 & 0,83 & $-1,87 / 1,54$ \\
\hline Cesáreas (n) & 1,38 & 0,98 & 1,32 & 0,45 & 0,46 & $0,45 / 0,48$ \\
\hline Hemorragia (ml) & $2.546,35$ & $1.265,60$ & $1.418,48$ & 559,15 & 0,005 & $577 / 1.578$ \\
\hline Hb previa (g/dl) & 12,23 & 2,07 & 11,21 & 1,71 & 0,11 & $-0,27 / 2,3$ \\
\hline $\mathrm{Hb}$ control* $(\mathrm{g} / \mathrm{dl})$ & 9,54 & 1,79 & 9,15 & 1,67 & 0,49 & $-0,76 / 1,55$ \\
\hline Tiempo Qx (min) & 128,08 & 29,82 & 99,48 & 25,98 & 0,74 & $12,82 / 44,86$ \\
\hline Días hospitalización & 5,62 & 4,81 & 7,92 & 6,83 & 0,10 & $5,50 / 0,90$ \\
\hline Días UCl & 0,81 & 1,65 & 0,60 & 1,32 & 0,37 & $-0,33 / 1,36$ \\
\hline Transfusiones (\# bolsas) & 3,73 & 2,80 & 2,16 & 1,77 & 0,01 & $0,24 / 2,89$ \\
\hline
\end{tabular}

Fuente: Archivo de Estadística Hospital General "Dr. Aurelio Valdivieso". Hb: hemoglobina. *Hemoglobina de control 12-14 horas. Tiempo Qx: tiempo quirúrgico reportado. UCl: Unidad de Cuidados Intensivos.

La necesidad de transfusiones sanguíneas se observó como una prioridad en la histerectomía obstétrica, el principal producto de transfusión fue el concentrado eritrocitario, sin embargo se observó la necesidad de transfundir otros productos, en específico 2 transfusiones de crioprecipitados, 3 de plaquetas y en 37 casos se requirió de plasma. Se presentaron 33 casos de hemorragia obstétrica masiva de los que 12 ocurrieron en el grupo de cirugía programada y 21 en el grupo de las urgencias.

\section{DISCUSIÓN}

La prevalencia reportada de $\mathrm{HO}$ en esta institución, así como las tasas de incidencia anual se encuentra ligeramente elevadas con respecto a países de Latinoamérica como el caso de Venezuela o Chile (1), donde se reportan incidencias de $0,07 \%$, hasta tres veces menor con respecto a las obtenidas en la presente revisión. Esto probablemente sea debido a que se trata de un hospital de concentración, el único resolutivo dentro del Estado lo que genera sesgo de referencia, por ser el único que cuenta con banco de sangre las 24 horas. Cabe reconocer que existe una muy alta tasa de cesáreas, rebasando el indicador del país que es de $40,9 \%$, que trae como consecuencia muchos casos de alteración en la adherencia placentaria, esta tuvo una prevalencia de 6,7/10.000 nacimientos, acercándose a la tendencia secular que presenta esta anomalía contemplando para el año 2020 de aproximadamente 1 caso por cada 1000 nacimientos (9). Con respecto a otras revisiones acerca de las alteraciones de la adherencia placentaria como principal patología que genera la realización de $\mathrm{HO}$, los reportes de México muestran una incidencia menor (8), debido a que la obtención proviene del total de consultas como divisor y no del total de procedimientos obstétricos, lo que puede generar una incidencia menor o mayor según se considere las consultas de primera vez o subsecuentes de obstetricia, por lo que se debe utilizar un mejor denominador como es el total de nacimientos.

Por lo que respecta al procedimiento de urgencia al compararlo con el programado, se puede observar en los resultados que la mayor dificultad técnica se presenta por la falta de programación y disponibilidad de medios para realizar el procedimiento en las mejores condiciones. Desde la óptica de que la mayoría de los casos son por alteraciones de la adherencia placentaria, a la que se asocian cambios vasculares importantes, es previsible que pueden presentar hemorragia mayor a la esperada, 
y requerir de transfusiones además de equipo especial en muchas ocasiones. Dentro de los procedimientos solo existió un caso de $\mathrm{HO}$ programada sin alteración en la adherencia placentaria que fue por sepsis. La frecuencia de presentación de hemorragia obstétrica masiva, mostró una mayor proporción de casos en el grupo de cirugía de urgencia con una razón de 1,75 veces, de manera que el realizar el procedimiento sin los medios necesarios expone a la paciente a riesgo innecesario.

Se observó además que la morbilidad por anemia es grave tanto por la cantidad de pacientes que terminan anémicas luego del procedimiento como por el alto consumo de hemoderivados que se tiene, pero una vez resuelto el problema hemorrágico la recuperación de la paciente es rápida y con poca morbilidad asociada a infecciones $u$ otro tipo de complicaciones, ya que el egreso se dio a los seis días promedio, en comparación con los resultados obtenidos en el estudio de Nava y cols (10) en el que se tiene estancia de once días con ingreso a la unidad de cuidados intensivos, sin embargo se asemeja en la proporción de pacientes que requirieron de la Unidad de Cuidados Intensivos, en el estudio citado fue de $42 \%$ de las pacientes, mientras que en nuestro caso el $47 \%$ de ellas requirió de esta unidad.

Una situación que no se debe pasar por alto es que más de la mitad (29 casos) de las indicaciones de $\mathrm{HO}$ fue resultado de la "obstetricia moderna" donde la alta tasa de cesáreas genera un incremento en los casos de alteraciones de la adherencia placentaria (acretismo), inserción anormal (placenta previa) o la necesidad de realizar una nueva intervención quirúrgica (cesárea iterativa), siendo estas indicaciones el $57 \%$ de las causas para realizar esta intervención. El estudio ecográfico realizado para diagnóstico en los casos de alteración de la adherencia placentaria basado en la escala de grises con los datos de lagos placentarios, pérdida de la zona de interfase útero-placentaria, adelgazamiento de miometrio y la presencia de protrusiones y engrosamientos a nivel vesical mostró una sensibilidad de $83 \%$ y valor predictivo positivo de $91 \%$, mientras que la especificidad y valor predictivo negativo fueron de $100 \%$, lo que indica que el estudio nos provee de gran información en cuanto a localización e inserción anómala de la placenta permitiendo que el procedimiento se realice en mejores condiciones mejorando sustancialmente los resultados.

Finalmente del comparativo realizado entre los grupos considerados en cuanto a cirugía de urgencia y cirugía programada, no se observaron diferencias importantes, salvo en el caso del menor tiempo quirúrgico para la cirugía programada que no alcanzó diferencias significativas, pero que permite con diagnóstico prenatal realizar un procedimiento más limpio y con menor riesgo de exposición de la paciente, con un menor sangrado que disminuye la necesidad de transfusiones sanguíneas y a su vez menor necesidad de ingreso a la Unidad de Cuidados Intensivos por encontrarse las pacientes en mejores condiciones hemodinámicas, si bien la comparación de esta variable no fue estadísticamente significativa.

\section{CONCLUSIONES}

La incidencia de $\mathrm{HO}$ en el Hospital General "Dr. Aurelio Valdivieso" SSO, es más alta con respecto a algunos países de Latinoamérica, teniendo como principal factor de riesgo el antecedente de cesárea, condición cada vez mayor en la práctica médica que ocasiona alteraciones en la placentación (placenta previa y alteración de la adherencia). El procedimiento quirúrgico es una condición de riesgo para anemia en el puerperio. El diagnóstico prenatal de adherencia anómala de la placenta por ultrasonografía mejora las condiciones del procedimiento quirúrgico al realizarlo de manera programada. Las mejores condiciones para la paciente ha generado que la mortalidad materna por hemorragia obstétrica masiva sea nula en los últimos tres años desde que se mejoró la técnica quirúrgica e implementó el programa código rojo para manejo de la emergencia obstétrica.

\section{REFERENCIAS}

1. Briceño-Pérez C, Briceño-Sanabria L, García S, Jaimes T, Briceño-Sanabria JC, Briceño-Sanabria C. Histerectomía obstétrica: análisis de 15 años. Rev Obstet Ginecol Venez 2009;69(2):89-96.

2. Flood KM, Said S, Geary M, Robson M, Fitzpatrick C, Malone FD. Changing trends in peripartum hysterectomy over the last 4 decades. Am J Obstet Gynecol 2009; 200(6):632.e1-6.

3. Wright JD, Pri-Paz S, Herzog TJ, Shah M, Bonanno C, Lewin SN, Simpson LL, Gaddipati S, Sun X, D'Alton ME, Devine P. Predictors of massive blood loss in women with placenta accreta. Am J Obstet Gynecol 2011;205(1):38.e1-6.

4. Reveles VJA, Villegas RG, Hernández HS, Grover PF, Hernández VCC, Patiño SA. Histerectomía obstétrica, incidencia, indicaciones y complicaciones. Ginecol Obstet Mex 2008;76(3):156-60.

5. Vélez-Álvarez GA, Agudelo-Jaramillo B, Gómez-Dávila JG, Zuleta-Tobón JJ. Código rojo: guía para el manejo de la hemorragia obstétrica. Rev Colomb Obstet 2009;60(1):34-48.

6. Briery $\mathrm{CM}$, Rose $\mathrm{CH}$, Hudson WT, Lutgendorf MA, Magann EF, Chauhan SP, Morrison JC. Planned vs emergent cesarean hysterectomy. Am J Obstet Gynecol 2007;197(2):154.e1-5.

7. Aguirre-Gas H. El error médico. Eventos adversos. Revista CONAMED 2005;11(4):36-41.

8. Objetivos de Desarrollo del Milenio 2015. México. Resumen Ejecutivo. Disponible en: http://200.23.8.225/ odm/Doctos/ResInfMex2015.pdf. 
9. INSP. Encuesta Nacional de Salud y Nutrición 2012. Evidencia para la política pública en salud 2013. Disponible en: http://ensanut.insp.mx/doctos/analiticos/ Cesareas.pdf.
10. Nava Flores J1, Paez Angulo JA, Veloz Martínez G, Sánchez Valle V, Hernández-Valencia M. [Indications and risk factors for emergency obstetric hysterectomy]. [Article in Spanish]. Ginecol Obstet Mex 2002;70:289-94. 